(1)

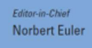
Journal of Nonlinear Mathematical Physics

\title{
Ermakov-Modulated Nonlinear Schrödinger Models. Integrable Reduction
}

Colin Rogers, Giuseppe Saccomandi, Luigi Vergori

To cite this article: Colin Rogers, Giuseppe Saccomandi, Luigi Vergori (2016) ErmakovModulated Nonlinear Schrödinger Models. Integrable Reduction, Journal of Nonlinear Mathematical Physics 23:1, 108-126, DOI:

https://doi.org/10.1080/14029251.2016.1135645

To link to this article: https://doi.org/10.1080/14029251.2016.1135645

Published online: 04 January 2021 


\title{
Ermakov-Modulated Nonlinear Schrödinger Models. Integrable Reduction
}

\author{
Colin Rogers \\ Australian Research Council Centre of Excellence for Mathematics \& Statistics of Complex Systems, School of \\ Mathematics, The University of New South Wales, Sydney, NSW2052, Australia. \\ c.rogers@unsw.edu.au \\ Giuseppe Saccomandi \\ Dipartimento di Ingegneria Industriale, Università degli Studi di Perugia, I-06125 Perugia, Italy. \\ School of Mathematics, Statistics and Applied Mathematics, National University of Ireland, Galway, University Road, \\ Galway, Ireland. \\ giuseppe.saccomandi@unipg.it \\ Luigi Vergori \\ School of Mathematics and Statistics, University of Glasgow, University Gardens 15 G128QW Glasgow, United \\ Kingdom. \\ luigi.vergori@glasgow.ac.uk
}

Received 1 September 2015

Accepted 1 October 2015

\begin{abstract}
Nonlinear Schrödinger equations with spatial modulation associated with integrable Hamiltonian systems of Ermakov-Ray-Reid type are introduced. An algorithmic procedure is presented which exploits invariants of motion to construct exact wave packet representations with potential applications in a wide range of physical contexts such as, 'inter alia', the analysis of Bloch wave and matter wave solitonic propagation and pulse transmission in Airy modulated NLS models. A particular Ermakov reduction for Mooney-Rivlin materials is set in the broader context of transverse wave propagation in a class of higher-order hyperelastic models of incompressible solids.
\end{abstract}

Keywords: Nonlinear Schödinger models; Ermakov reduction; Nonlinear waves.

2010 Mathematics Subject Classification: 34C20, 35Q55, 35Q74

\section{Introduction}

Spatially modulated equations arise classically in a range of important physical contexts, such as in the analysis of crack and contact problems in inhomogeneous elastic media. Analytic solution of such problems may, in particular, be obtained for appropriate inhomogeneities via a termination of Bergman series approach as described in Refs. [11, 12, 34]. In elastodynamics and visco-elastodynamics an analogous procedure involves truncation of wave front expansions such as were investigated by Karal and Keller [24]. Exact solution may thereby be obtained to a variety of initial boundary value problems for inhomogeneous elastic and viscoelastic media (see e.g. Refs. [2,27,31,32]). In Ref. [2] connection was made between termination of wave front expansions and the iterated action of Bäcklund-Darboux type transformations. This termination of Bergman series approach has also been applied in the setting of electromagnetic wave propagation in nonlinear dielectric media [33] to isolate $\mathbf{D}(\mathbf{E})$ and $\mathbf{B}(\mathbf{H})$ constitutive laws for which associated 'modulation' hodograph systems admit integration. In a nonlinear elastodynamic context, an analogous 
procedure may be used to isolate the constitutive laws introduced by Cekirge and Varley in Ref. [8] and used therein to approximate loading response in a range of materials described by model nonlinear stress-strain laws.

Modulated nonlinear Schrödinger models equations arise most notably in an optics setting, and have an extensive literature (see e.g. Refs. [3, 4, 50,52-54] and work cited therein). Such models are of importance, in particular, in the area of solitonic management (Malomed [26]). Soliton and large amplitude Bloch wave propagation in optical lattices have been investigated via a modulated nonlinear Schrödinger (NLS) model with external potential by Zhang et al [52]. In that work, a procedure previously introduced by Belmonte-Beitia et al $[3,4]$ was adopted wherein the modulation is driven by the classical Ermakov equation. The latter admits a nonlinear superposition principle and classes of exact solutions relevant to Bloch wave propagation were generated thereby in terms of Mathieu functions.

Here, it is established that the procedure in Ref. [52] may be extended and embedded in an algorithmic procedure wherein the modulation is driven by an integrable system of Ermakov-RayReid type. The latter system, which includes the classical Ermakov equation as a particular reduction, likewise admits nonlinear superposition principles generated via a characteristic invariant of motion.

Nonlinear coupled systems of Ermakov-Ray-Reid type have their roots in the classical work of Ermakov [18] and were introduced by Ray and Reid [29,30]. Subsequently, 2+1-dimensional Ermakov-Ray-Reid systems were constructed in Ref. [36] and extensions to arbitrary order and dimension which preserve the characteristic Ray-Reid invariants were presented in Ref. [47]. Multicomponent Ermakov-Ray-Reid systems were derived in the physical context of N-layer hydrodynamic systems in Ref. [37].

The occurrence of Ermakov-Ray-Reid systems in a nonlinear optics context is well-documented (see e.g. Refs. [13, 19-22, 38, 51]). Such systems have been derived, notably, to describe the evolution of the size and shape of the light spot and wave front in elliptic Gaussian beams [13,22]. In Ref. [43], a variational approximation approach was adopted to obtain reduction of a class of modulated 3+1-dimensional NLS equations to integrable Hamiltonian systems of Ermakov-Ray-Reid type for the beam widths. These modulated NLS equations incorporated a logarithmic potential such as was proposed by Snyder and Mitchell [49] in an analysis of the propagation of Gaussian beams in a saturable medium. A logarithmic nonlinear Schrödinger equation was originally set down by Bialynicki-Birula and Mycielski $[5,6]$ in a nonlinear wave mechanics context and its noteworthy for its admittance of gausson-type solutions [7,45]. The dynamics of the propagation of partially coherent beams has been investigated via a logarithmic nonlinear media model by Królikowski et al [25] while solitonic interaction in such media has been analysed by Christodoulis et al [10] and Hansen et al [23]. Integrable Hamiltonian reductions of Ermakov-Ray-Reid type for 2+1-dimensional hydrodynamic Madelung systems incorporating modulated logarithmic terms together with de-Broglie-Bohm quantum potential have been obtained via an elliptic vortex representation in Ref. [40]. Indeed, integrable Ermakov-Ray-Reid systems have in recent years been shown to arise in a wide range of important physical contexts, including warm-core oceanographic elliptic eddy theory, rotating shallow water hydrodynamics, anisentropic gasdynamics and magnetogasdynamics $[39,41,42,48]$. They also arise in a spiralling elliptic soliton model of Ref. [17] and its extension in the Bose-Einstein setting in Ref. [1]. The Ermakov-Ray-Reid connections in the latter cases are demonstrated in Ref. [43]. 
Here, a class of NLS model equations are investigated wherein the spatial modulation of the nonlinear term is driven by an integrable Ermakov-Ray-Reid system with its characteristic invariant of motion. Attention is initially restricted to such systems with underlying Hamiltonian structure and the two key invariants are then used in an algorithmic procedure for the construction of a class of exact wave packet representations for the 'Ermakov-modulated' NLS equations. The method is illustrated by application, both to a modulated NLS equation relevant to the analysis of Bloch wave propagation as investigated in Ref. [52] and to a NLS equation with Airy-type spatial modulation.

To conclude, a classical Ermakov reduction for a Mooney-Rivlin material is derived via a wave packet ansatz in the wider setting of propagation in higher-order hyperelastic models of incompressible solids. It is noted that Destrade and Saccomandi [16] recently derived a Ermakov reduction in an investigation of wave propagation in a neo-Hookean material that is stretched linearly with time.

\section{Integrable Ermakov-Ray-Reid Reduction}

Here, we consider the class of spatially modulated nonlinear Schrödinger (NLS) equations

$$
i U_{t}+U_{x x}-\left[\Omega(x)+\Lambda(x)|U|^{2}\right] U=0,
$$

where $\Omega(x)$ is an external potential and $\Lambda(x)$ is a modulation of the nonlinear term. Introducing the wave packet representation

$$
U=q(x) \exp [i(r(x)-\omega t)]
$$

$\omega$ being a constant, into (2.1) and separating the real and imaginary parts lead to the equations for the amplitude $q$ and the phase $r$, namely

$$
\begin{gathered}
\ddot{q}+\omega q-\dot{r}^{2} q-\left[\Omega(x)+\Lambda(x) q^{2}\right] q=0, \\
2 \dot{r} \dot{q}+\ddot{r} q=0,
\end{gathered}
$$

where a dot indicates a derivative with respect to the spatial variable $x$. Integrating (2.4) gives

$$
\dot{r}=\frac{k_{\mathrm{I}}}{q^{2}},
$$

where $k_{\mathrm{I}}$ is an arbitrary constant of integration.

In the sequel, the modulation $\Lambda(x)$ is assumed to be of the form

$$
\Lambda(x)=\frac{1}{\rho^{6}} H\left(\frac{\rho}{q}\right),
$$

where $\rho=\rho(x)$ satisfies the equation

$$
\ddot{\rho}+[\omega-\Omega(x)] \rho=\frac{1}{\rho^{2} q} \Psi\left(\frac{q}{\rho}\right)
$$

and $H$ and $\Psi$ are arbitrary functions. Thus, inserting (2.5) and (2.6) into (2.3) yields the equation

$$
\ddot{q}+[\omega-\Omega(x)] q=\frac{1}{q^{2} \rho} \Phi\left(\frac{\rho}{q}\right)
$$


where

$$
\Phi\left(\frac{\rho}{q}\right) \equiv\left(\frac{\rho}{q}\right)^{-5} H\left(\frac{\rho}{q}\right)+k_{\mathrm{I}}^{2} \frac{\rho}{q} .
$$

The coupled pair of nonlinear equations (2.7) and (2.8) constitute a Ermakov-Ray-Reid system [29, 30, 36, 37, 47]. On introduction of new dependent and independent variables $\alpha, \beta$ and $\zeta$ according to

$$
q=\alpha(\zeta) \phi, \quad \rho=\beta(\zeta) \phi, \quad \zeta=\psi / \phi,
$$

where $\phi$, and $\psi$ are linearly independent solutions with unit Wronskian $\phi \dot{\psi}-\dot{\phi} \psi$ of the base equation

$$
\ddot{\mathscr{R}}+[\omega-\Omega(x)] \mathscr{R}=0,
$$

it is seen that the Ermakov-Ray-Reid system reduces to the autonomous canonical form

$$
\alpha^{\prime \prime}=\frac{1}{\alpha^{2} \beta} \Phi(\beta / \alpha), \quad \beta^{\prime \prime}=\frac{1}{\beta^{2} \alpha} \Psi(\alpha / \beta),
$$

where the prime denotes a derivative with respect to $\zeta$. This system admits the characteristic Ermakov-type invariant

$$
\mathscr{I}=\frac{1}{2}\left(\alpha \beta^{\prime}-\beta \alpha^{\prime}\right)^{2}+\int^{\sigma=\beta / \alpha} \Phi(\sigma) d \sigma+\int^{\tau=\alpha / \beta} \Psi(\tau) d \tau .
$$

If, in addition,

$$
\Psi(\tau)-\Phi(\sigma)=\tau \frac{\mathrm{d} \Psi}{\mathrm{d} \tau}(\tau)-\sigma \frac{\mathrm{d} \Phi}{\mathrm{d} \sigma}(\sigma)
$$

system (2.12) is Hamiltonian $[38,43]$ with

$$
\alpha^{\prime \prime}=-\frac{\partial V}{\partial \alpha}, \quad \beta^{\prime \prime}=-\frac{\partial V}{\partial \beta}, \quad V=\frac{1}{\alpha^{2}} J(\beta / \alpha), \quad J(\beta / \alpha)=\frac{\alpha}{2 \beta}[\Phi(\beta / \alpha)+\Psi(\alpha / \beta)] .
$$

Then (2.12) admits the parametrization

$$
\alpha^{\prime \prime}=\frac{1}{\alpha^{3}}\left[2 J(\sigma)+\sigma \frac{\mathrm{d} J}{\mathrm{~d} \sigma}(\sigma)\right], \quad \beta^{\prime \prime}=-\frac{1}{\alpha^{3}} \frac{\mathrm{d} J}{\mathrm{~d} \sigma}(\sigma),
$$

with associated Hamiltonian

$$
\mathscr{H}=\frac{1}{2}\left(\alpha^{\prime 2}+\beta^{\prime 2}\right)+\frac{1}{\alpha^{2}} J(\beta / \alpha) .
$$

The Ermakov invariant becomes

$$
\mathscr{I}=\frac{1}{2}\left(\alpha \beta^{\prime}-\alpha^{\prime} \beta\right)^{2}+\frac{\alpha^{2}+\beta^{2}}{\alpha^{2}} J(\beta / \alpha) .
$$

This pair of invariant relations in $\mathscr{I}$ and $\mathscr{H}$ may now be adduced to solve the canonical autonomous Ermakov-Ray-Reid system (2.16) for $\alpha, \beta$ via the algorithmic procedure introduced in Refs. [38, 
43]. Thus, application of the identity

$$
\left(\alpha^{2}+\beta^{2}\right)\left(\alpha^{\prime 2}+\beta^{\prime 2}\right)-\left(\alpha \beta^{\prime}-\alpha^{\prime} \beta\right)^{2} \equiv\left(\alpha \alpha^{\prime}+\beta \beta^{\prime}\right)^{2}
$$

together with the pair of invariant relations (2.17), (2.18) shows that

$$
2 \Sigma\left[\mathscr{H}-\frac{1}{\alpha^{2}} J(\beta / \alpha)\right]-2\left[\mathscr{I}-\frac{\Sigma}{\alpha^{2}} J(\beta / \alpha)\right]=\frac{1}{4} \Sigma^{\prime 2}
$$

where $\Sigma=\alpha^{2}+\beta^{2}$. Reduction of (2.20) yields

$$
\frac{1}{8} \Sigma^{\prime 2}=\mathscr{H} \Sigma-\mathscr{I}
$$

Clearly, equation (2.21) does not admit a solution if $\mathscr{H}<0$ and $\mathscr{I} \geq 0$, while, on integration,

$$
\Sigma=\frac{1}{\mathscr{H}}\left[\mathscr{I}+2 \mathscr{H}^{2}\left(\zeta-\zeta_{0}\right)^{2}\right]
$$

$\zeta_{0}$ being an integration constant, if $\mathscr{H}>0$ or $\mathscr{H}<0$ and $\mathscr{I} \leq 0$. If $\mathscr{H}=0$ and $\mathscr{I} \leq 0$

$$
\Sigma=2 \sqrt{2|\mathscr{I}|} \zeta+\zeta_{0}
$$

Now,

$$
\frac{\mathrm{d}}{\mathrm{d} \zeta} \arctan \sigma=\frac{\alpha \beta^{\prime}-\alpha^{\prime} \beta}{\Sigma}
$$

so that, on introduction of the new independent variable $\tilde{\zeta}$ according to

$$
\mathrm{d} \tilde{\zeta}=\Sigma^{-1} \mathrm{~d} \zeta
$$

the invariant relation (2.18) shows that $\sigma=\beta / \alpha$ is given via the constant of motion expression

$$
\mathscr{I}=\frac{1}{2}\left(\frac{\mathrm{d}}{\mathrm{d} \tilde{\zeta}} \arctan \sigma\right)^{2}+\left(1+\sigma^{2}\right) J(\sigma)
$$

whence

$$
\frac{1}{\sqrt{2}} \int \frac{d \sigma}{\left(1+\sigma^{2}\right) \sqrt{\mathscr{I}-\left(1+\sigma^{2}\right) J(\sigma)}}=\tilde{\zeta}+C
$$


where, by virtue of (2.22) and (2.25), up to an additive constant,

$$
\tilde{\zeta}= \begin{cases}\frac{1}{\sqrt{2 \mathscr{I}}} \arctan \left[\mathscr{H} \sqrt{\frac{2}{\mathscr{I}}}\left(\zeta-\zeta_{0}\right)\right], & \text { if } \mathscr{H}>0, \mathscr{I}>0, \\ -\frac{1}{2 \mathscr{H}\left(\zeta-\zeta_{0}\right)}, & \text { if } \mathscr{H}>0, \mathscr{I}=0, \\ -\frac{\zeta}{\zeta_{0}}, & \text { if } \mathscr{H}=0, \mathscr{I}=0 \\ \frac{\operatorname{sign}(\mathscr{H})}{\sqrt{2|\mathscr{I}|}} \operatorname{arctanh}\left[|\mathscr{H}| \sqrt{\frac{2}{|\mathscr{I}|}}\left(\zeta-\zeta_{0}\right)\right], & \text { if } \mathscr{H} \neq 0, \mathscr{I}<0 \\ \frac{1}{\sqrt{2|\mathscr{I}|}} \ln \sqrt{\left|\zeta-\zeta_{0}\right|}, & \text { if } \mathscr{H}=0, \mathscr{I}<0\end{cases}
$$

The Ermakov variables $\alpha$ and $\beta$ in the autonomised system are given in terms of $\Sigma$ and $\sigma$ by the relations

$$
\alpha= \pm \sqrt{\frac{\Sigma}{1+\sigma^{2}}}, \quad \beta= \pm \sigma \sqrt{\frac{\Sigma}{1+\sigma^{2}}}
$$

In particular, if

$$
J=\frac{\varepsilon}{1+\sigma^{2}} \quad(\varepsilon>0),
$$

the Ermakov-Ray-Reid system (2.16) adopts the symmetric form

$$
\alpha^{\prime \prime}=\frac{2 \varepsilon \alpha}{\left(\alpha^{2}+\beta^{2}\right)^{2}}, \quad \beta^{\prime \prime}=\frac{2 \varepsilon \beta}{\left(\alpha^{2}+\beta^{2}\right)^{2}},
$$

and corresponds to a special case in which the Wronskian in the Ermakov invariant relation (2.18) is constant. The relations (2.27) and (2.28) then together show that

$$
\sigma=\tan \left\{\sqrt{1-\frac{\varepsilon}{\mathscr{I}}} \arctan \left[\mathscr{H} \sqrt{\frac{2}{\mathscr{I}}}\left(\zeta-\zeta_{0}\right)\right]+\bar{C}\right\}
$$

where $\bar{C}$ is an arbitrary constant of integration.

In a more general situation wherein $J(\sigma)$ is such that

$$
\mathscr{I}-\left(1+\sigma^{2}\right) J=\bar{\alpha}+\frac{\bar{\beta}}{1+\sigma^{2}}+\frac{\bar{\gamma}}{\left(1+\sigma^{2}\right)^{2}}+\bar{\delta}\left(1+\sigma^{2}\right)
$$

an elliptic integral representation for $\sigma$ may be obtained directly via

$$
\left(\frac{\mathrm{d} \sigma}{\mathrm{d} \tilde{\zeta}}\right)^{2}=2\left[\bar{\alpha}\left(1+\sigma^{2}\right)^{2}+\bar{\beta}\left(1+\sigma^{2}\right)+\bar{\gamma}+\bar{\delta}\left(1+\sigma^{2}\right)^{3}\right] .
$$

Various specialisation of (2.34) have previously led to results of physical interest in a wide range of physical contexts with nonlinear Schrödinger-type models. In particular, recently, in Ref. [9] in 
the context of dark soliton pulse propagation modelled by a nonic NLS model equation, it was recorded that the nonlinear equation

$$
\begin{aligned}
\left(\frac{\mathrm{d} \sigma}{\mathrm{d} \tilde{\zeta}}\right)^{2}= & -A_{0}^{2}-\left(3 b+k^{2}-2\right) \sigma^{2} \\
& -\left[3 b^{2}+2\left(k^{2}-2\right) b+1-k^{2}\right] \sigma^{4} / A_{0}^{2} \\
& -\left[b^{3}+\left(k^{2}-2\right) b^{2}+\left(1-k^{2}\right) b\right] \sigma^{6} / A_{0}^{4} .
\end{aligned}
$$

admits the two-parameter class of exact solutions

$$
\sigma=\frac{A_{0}}{\sqrt{\operatorname{dn}^{2}(\tilde{\zeta}, k)+b}},
$$

where $\tilde{\zeta}$ is given in terms $\zeta$ by (2.28). Here, the alignment of (2.34) with (2.35) requires that the constant $\bar{\alpha}, \bar{\beta}, \bar{\gamma}$ and $\bar{\delta}$ be such that

$$
\begin{aligned}
& \bar{\alpha}=\frac{3\left[b^{3}+\left(k^{2}-2\right) b^{2}+\left(1-k^{2}\right) b\right]}{2 A_{0}^{4}}-\frac{3 b^{2}+2\left(k^{2}-2\right) b+1-k^{2}}{2 A_{0}^{2}} \\
& \bar{\beta}=-\frac{3 b+k^{2}-2}{2}+\frac{3 b^{2}+2\left(k^{2}-2\right) b+1-k^{2}}{A_{0}^{2}}-\frac{3\left[b^{3}+\left(k^{2}-2\right) b^{2}+\left(1-k^{2}\right) b\right]}{2 A_{0}^{4}} \\
& \bar{\gamma}=-\frac{A_{0}^{2}}{2}+\frac{3 b+k^{2}-2}{2}-\frac{3 b^{2}+2\left(k^{2}-2\right) b+1-k^{2}}{2 A_{0}^{2}}+\frac{b^{3}+\left(k^{2}-2\right) b^{2}+\left(1-k^{2}\right) b}{2 A_{0}^{4}} \\
& \bar{\delta}=-\frac{b^{3}+\left(k^{2}-2\right) b^{2}+\left(1-k^{2}\right) b}{2 A_{0}^{4}}
\end{aligned}
$$

The corresponding solutions $\alpha, \beta$ of the autonomous Ermakov-Ray-Reid system (2.16) with $J(\Delta)$ given by (2.33) are determined in terms of $\Sigma$ and $\sigma$ by the relations (2.29). For the sake of brevity, we shall henceforth limit our analysis to the case $\mathscr{H}>0$ and $\mathscr{I}>0$. Then, from (2.22), (2.28) ${ }_{1}$ and (2.29) we deduce that

$$
\begin{aligned}
& \alpha= \pm \sqrt{\frac{\left[\mathscr{I}+2 \mathscr{H}^{2}\left(\zeta-\zeta_{0}\right)^{2}\right]\left[\operatorname{dn}^{2}(\tilde{\zeta}, k)+b\right]}{\mathscr{H}\left[A_{0}^{2}+\operatorname{dn}^{2}(\tilde{\zeta}, k)+b\right]}}, \\
& \beta= \pm A_{0} \sqrt{\frac{\mathscr{I}+2 \mathscr{H}^{2}\left(\zeta-\zeta_{0}\right)^{2}}{\mathscr{H}\left[A_{0}^{2}+\operatorname{dn}^{2}(\tilde{\zeta}, k)+b\right]}} .
\end{aligned}
$$

The spatial modulation term $\Lambda(x)$, by virtue of (2.6) and (2.9) is given by

$$
\Lambda(x)=\frac{1}{\rho^{6}}\left[\left(\frac{\rho}{q}\right)^{5} \Phi\left(\frac{\rho}{q}\right)-k_{\mathrm{I}}^{2}\left(\frac{\rho}{q}\right)^{6}\right],
$$

where, for the present class of Ermakov-Ray-Reid systems (2.16) with underlying Hamiltonian structure, it is seen that

$$
\Phi\left(\frac{\rho}{q}\right)=\Phi\left(\frac{\beta}{\alpha}\right)=\Phi(\sigma)=\frac{\mathrm{d}}{\mathrm{d} \sigma}\left[\sigma^{2} J(\sigma)\right]
$$


The original Ermakov variables $q$ and $\rho$ as given by the relations (2.10) have dependence on the pair of linearly independence solutions $\phi, \psi$ with unit Wronskian of the base equation (2.11) which, in turn, depends on the nature of the external potential $\Omega$.

In the absence of an external potential $\Omega$, if $\omega=\lambda^{2}>0$, then (2.11) admits the pair of linearly independent solutions

$$
\phi=\cos (\lambda x), \quad \psi=\sin (\lambda x)
$$

with unit Wronskian. Accordingly, $q$ and $\rho$ admit the representations

$$
q=\alpha(\zeta) \cos (\lambda x), \quad \rho=\beta(\zeta) \cos (\lambda x)
$$

where $\zeta=\tan (\lambda x)$ and the Ermakov variables $\alpha$ and $\beta$ are given by the expressions (2.38).

In the case of non-zero $\Omega(x)$, the appropriate representation for this external potential depends on the physical context. In the sequel, two cases of potential interest are summarised.

\subsection{Bloch Wave Propagation}

In Ref. [52], Zhang et al adopted the external potential

$$
\Omega(x)=2 V_{0} \cos 2 x
$$

in the context of modulated Bloch wave propagation in optical lattices. In this case, Eq. (2.11) admits the pair of linearly independent solutions

$$
\phi=\text { Mathieu } \mathrm{C}\left(\omega, V_{0}, x\right), \quad \psi=\text { Mathieu } \mathrm{S}\left(\omega, V_{0}, x\right),
$$

with unit Wronskian. Accordingly here, in explicit terms

$$
\begin{aligned}
& q= \pm \text { Mathieu } \mathrm{C}\left(\omega, V_{0}, x\right) \sqrt{\frac{\left[\mathscr{I}+2 \mathscr{H}^{2}\left(\zeta-\zeta_{0}\right)^{2}\right]\left[\mathrm{dn}^{2}(\tilde{\zeta}, k)+b\right]}{\mathscr{H}\left[A_{0}^{2}+\mathrm{dn}^{2}(\tilde{\zeta}, k)+b\right]}} \\
& \rho= \pm A_{0} \text { Mathieu } \mathrm{C}\left(\omega, V_{0}, x\right) \sqrt{\frac{\mathscr{I}+2 \mathscr{H}^{2}\left(\tilde{\zeta}-\zeta_{0}\right)^{2}}{\mathscr{H}\left[A_{0}^{2}+\operatorname{dn}^{2}(\tilde{\zeta}, k)+b\right]}}
\end{aligned}
$$

where $\tilde{\zeta}$ is given by $(2.28)_{1}$ with

$$
\zeta=\frac{\text { Mathieu } \mathrm{S}\left(\omega, V_{0}, x\right)}{\text { Mathieu } \mathrm{C}\left(\omega, V_{0}, x\right)}
$$

\subsection{Airy-Jacobi Elliptic Modulation}

Bessel-type modulation of the nonlinear Schrödinger equation has recently been investigated in a nonlinear optics setting by Zhong et al [54] via a similar reduction. Bright and dark solitary wave solutions were generated thereby. Here, we consider Airy dependent spatial modulation. 
Thus, the external potential

$$
\Omega(x)=\Omega_{0} x
$$

is posited in the modulated NLS equation (2.1) so that the linear base equation (2.11) of the present Ermakov reduction procedure becomes the Airy equation

$$
\frac{\mathrm{d}^{2} \mathscr{A}}{\mathrm{d} \tilde{x}^{2}}-\tilde{x} \mathscr{A}=0,
$$

where

$$
\tilde{x}=\Omega_{0}^{1 / 3}\left(x-\omega \Omega_{0}^{-1}\right) .
$$

The canonical reduction (2.48) admits a pair of linearly independent solutions in terms of modified Bessel functions, or alternatively, in terms of Airy functions $\operatorname{Ai}(\tilde{x}), \operatorname{Bi}(\tilde{x})$. If we proceed with the latter representation, $\phi=\operatorname{Ai}(\tilde{x})$ and $\psi=\pi \operatorname{Bi}(\tilde{x})$ are independent solutions to (2.48) with unit Wronskian and thus the autonomisation relations (2.10) become

$$
q=\operatorname{Ai}(\tilde{x}) \alpha(\zeta), \quad \rho=\operatorname{Ai}(\tilde{x}) \beta(\zeta), \quad \zeta=\pi \frac{\operatorname{Bi}(\tilde{x})}{\operatorname{Ai}(\tilde{x})}
$$

It is recalled that the variables $\alpha$ and $\beta$ in (2.50) are here determined by the Hamiltonian Ermakov-Ray-Reid system (2.16) and are given in terms of $\Sigma$ and $\sigma$ by the relations (2.29). It is noted that $\Sigma$ is independent of $J(\beta / \alpha)$ and is expressed in terms of $\zeta$ by (2.22).

Here, to emphasise the scope of the reduction procedure, we record the solution for $q$ and $\rho$ corresponding to another basic class of solutions for $\sigma=\beta / \alpha$ in terms of Jacobi elliptic functions. Thus, the two-parameter specialisation

$$
\left(\frac{\mathrm{d} \sigma}{\mathrm{d} \tilde{\zeta}}\right)^{2}=-\tilde{a} \sigma^{4}+\tilde{b} \sigma^{2}+\tilde{c}
$$

with

$$
\bar{\alpha}=-\frac{\tilde{a}}{2}, \quad \bar{\beta}=\frac{2 \tilde{a}+\tilde{b}}{2}, \quad \bar{\gamma}=\frac{\tilde{c}-(\tilde{a}+\tilde{b})}{2}, \quad \bar{\delta}=0,
$$

is considered.

This reduction was used in Ref. [44] in connection with the application of a variant of the present algorithmic procedure to an unmodulated cubic-quintic NLS equation incorporating both a de Broglie-Bohm quantum potential and a generalised Kaup-Newell type nonlinear dispersion term.

If $\tilde{a}$ and $\tilde{c}$ are positive, then the explicit exact solution of (2.51) can be written in terms of the Jacobi elliptic function cn as

$$
\sigma=\sigma_{0} \operatorname{cn}(s \tilde{\zeta}, k)
$$

where

$$
\sigma_{0}=\sqrt{\frac{\tilde{b}+\sqrt{4 \tilde{a} \tilde{c}+\tilde{b}^{2}}}{2 \tilde{a}}}, \quad s=\sqrt[4]{4 \tilde{a} \tilde{c}+\tilde{b}^{2}}, \quad k=\frac{\sqrt{\tilde{a}} \sigma_{0}}{s} .
$$


Thus, in this case, the original Ermakov variables $\rho, q$ are given by

$$
\begin{aligned}
& q= \pm \operatorname{Ai}(\tilde{x}) \sqrt{\frac{\mathscr{I}+2 \mathscr{H}^{2}\left(\zeta-\zeta_{0}\right)^{2}}{\mathscr{H}\left[1+\sigma_{0}^{2} \mathrm{cn}^{2}(\tilde{\zeta}, k)\right]}}, \\
& \rho= \pm A_{0} \operatorname{Ai}(\tilde{x}) \operatorname{cn}(s \tilde{\zeta}, k) \sqrt{\frac{\mathscr{I}+2 \mathscr{H}^{2}\left(\zeta-\zeta_{0}\right)^{2}}{\mathscr{H}\left[1+\sigma_{0}^{2} \mathrm{cn}^{2}(s \tilde{\zeta}, k)\right]}},
\end{aligned}
$$

where $\tilde{x}$ is given in terms of $x$ by the linear relation (2.49), while $\tilde{\zeta}$ is determined via $(2.28)_{1}$ and the relation $(2.50)_{3}$. The modulation $\Lambda(x)$ is obtained via the relations (2.39) and (2.40) with

$$
J\left(\frac{\beta}{\alpha}\right) \equiv J(\sigma)=\frac{1}{\left(1+\sigma^{2}\right)}\left[\mathscr{I}+\frac{\tilde{a}}{2}-\frac{\tilde{b}+2 \tilde{a}}{2\left(1+\sigma^{2}\right)}-\frac{\tilde{c}-(\tilde{a}+\tilde{b})}{2\left(1+\sigma^{2}\right)^{2}}\right] .
$$

\section{A Classical Ermakov-Type Reduction}

Insertion of the relation (2.5) into (2.3) produces the wave amplitude equation, viz

$$
q_{x x}+\left[\omega-\Omega(x)-\Lambda(x) q^{2}\right] q=\frac{k_{\mathrm{I}}^{2}}{q^{3}},
$$

and, on change of dependent and independent variables according to

$$
\alpha=q / \rho, \quad \mathrm{d} x^{*}=\rho^{-2} \mathrm{~d} x,
$$

this becomes

$$
\alpha_{x^{*} x^{*}}+\left[\frac{\rho_{x x}}{\rho}+\omega-\Omega(x)-\Lambda(x) \rho^{2} \alpha^{2}\right] \rho^{4} \alpha=\frac{k_{\mathrm{I}}^{2}}{\alpha^{3}} .
$$

If it is now required that

$$
\Lambda(x)=-\frac{\lambda}{\rho^{6}},
$$

where $\lambda$ is a constant and $\rho$ is governed by the classical Ermakov equation

$$
\rho_{x x}+[\omega-\Omega(x)] \rho=\frac{c_{\mathrm{I}}}{\rho^{3}},
$$

$c_{\mathrm{I}}$ being an arbitrary constant. Then the Ermakov-modulated wave amplitude $\alpha$ is given by an autonomous equation

$$
\alpha_{x^{*} x^{*}}+c_{\mathrm{I}} \alpha+\lambda \alpha^{3}=\frac{k_{\mathrm{I}}^{2}}{\alpha^{3}} .
$$

A single integration of (3.6) yields

$$
\alpha_{x^{*}}^{2}+c_{\mathrm{I}} \alpha^{2}+\frac{\lambda}{2} \alpha^{4}+\frac{k_{\mathrm{I}}^{2}}{\alpha^{2}}=k_{\mathrm{II}}
$$

where $k_{\text {II }}$ is an integration constant, and, on setting $\mathscr{A}=\alpha^{2}$, it is seen that

$$
\frac{1}{4} \mathscr{A}_{x^{*}}^{2}=k_{\mathrm{II}} \mathscr{A}-\frac{\lambda}{2} \mathscr{A}^{3}-c_{\mathrm{I}} \mathscr{A}^{2}-k_{\mathrm{I}}^{2} \equiv \mathscr{B}(\mathscr{A}) .
$$

A qualitative analysis à la Weierstrass of equation (3.8) reveals that it admits non singular solutions only in the following cases 


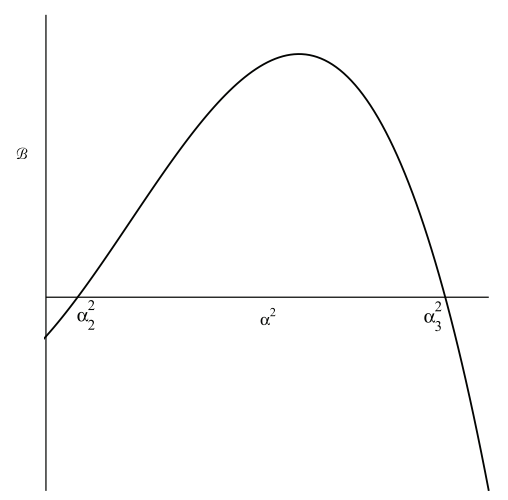

(a) Cases (i) and (ii).

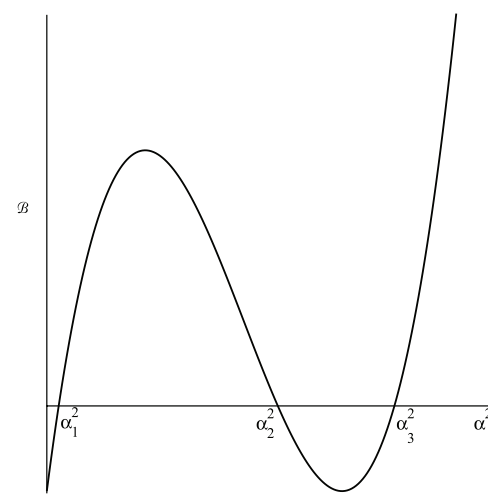

(b) Case (iii).

Fig. 1. The different profiles of the function $\mathscr{B}$ guaranteeing the existence of non singular solutions to equations (3.7) and (3.8) with modulation $\Lambda$ as in (3.4) $(\lambda \neq 0)$.

(i) $\lambda>0, c_{\mathrm{I}} \leq 0, k_{\mathrm{II}} \geq-c_{\mathrm{I}}^{2} /(2 \lambda)$ and

$$
k_{\mathrm{I}}^{2} \leq \frac{8 \delta\left(\sqrt{\delta}-c_{\mathrm{I}}\right)-6 \lambda c_{\mathrm{I}} k_{\mathrm{II}}}{27 \lambda^{2}}
$$

where $\delta=c_{\mathrm{I}}^{2}+3 \lambda k_{\mathrm{II}} / 2$;

(ii) $\lambda>0, c_{\mathrm{I}}>0, k_{\mathrm{II}}>0$ and $k_{\mathrm{I}}$ satisfying inequality (3.9);

(iii) $\lambda<0, c_{\mathrm{I}}>0,0<k_{\mathrm{II}}<2 c_{\mathrm{I}}^{2} /(3|\lambda|)$ and

$$
\frac{6|\lambda| c_{\mathrm{I}} k_{\mathrm{II}}-8 \delta\left(\sqrt{\delta}+c_{\mathrm{I}}\right)}{27 \lambda^{2}} \leq k_{\mathrm{I}}^{2}<\frac{6|\lambda| c_{\mathrm{I}} k_{\mathrm{II}}+8 \delta\left(\sqrt{\delta}-c_{\mathrm{I}}\right)}{27 \lambda^{2}}
$$

(iv) $\lambda=0, c_{\mathrm{I}}>0, k_{\mathrm{II}}>0, k_{\mathrm{I}}^{2} \leq k_{\mathrm{II}}^{2} /\left(4 c_{\mathrm{I}}\right)$.

Setting

$$
\alpha_{n}^{2}=\left|\frac{4 \sqrt{\delta}}{3|\lambda|} \cos \frac{\vartheta+2 n \pi}{3}-\frac{2 c_{\mathrm{I}}}{3 \lambda}\right| \quad(n=1,2,3)
$$

with

$$
\vartheta=\arccos \left[-\operatorname{sign}(\lambda) \frac{27 \lambda^{2} k_{\mathrm{I}}^{2}+18 \lambda c_{\mathrm{I}} k_{\mathrm{II}}+8 c_{\mathrm{I}}^{3}}{8 \delta \sqrt{\delta}}\right]
$$

by using Cardano's formula one can readily check that $\alpha_{2}^{2}$ and $\alpha_{3}^{2}\left(\alpha_{2}^{2} \leq \alpha_{3}^{2}\right)$ are the (positive) zeroes of $\mathscr{B}$ in cases $(i)$ and (ii), while $\alpha_{1}^{2}<\alpha_{2}^{2} \leq \alpha_{3}^{2}(n=1,2,3)$ are the zeroes of $\mathscr{B}$ in case (iii). It can be also proved that the solution to (3.7) is

$$
\alpha=\sqrt{\alpha_{3}^{2}-\left(\alpha_{3}^{2}-\alpha_{2}^{2}\right) \operatorname{sn}^{2}\left[\sqrt{\frac{\lambda\left(\alpha_{1}^{2}+\alpha_{3}^{2}\right)}{2}}\left(x^{*}-x_{0}^{*}\right), \sqrt{\frac{\alpha_{3}^{2}-\alpha_{2}^{2}}{\alpha_{1}^{2}+\alpha_{3}^{2}}}\right]}
$$


in the former cases and

$$
\alpha=\sqrt{\alpha_{1}^{2}+\left(\alpha_{2}^{2}-\alpha_{1}^{2}\right) \operatorname{sn}^{2}\left[\sqrt{\frac{|\lambda|\left(\alpha_{3}^{2}-\alpha_{1}^{2}\right)}{2}}\left(x^{*}-x_{0}^{*}\right), \sqrt{\frac{\alpha_{2}^{2}-\alpha_{1}^{2}}{\alpha_{3}^{2}-\alpha_{1}^{2}}}\right]}
$$

in the latter. In the absence of modulation, i.e., $\lambda=0$, the solution to (3.7) is instead

$$
\alpha=\sqrt{\frac{k_{\mathrm{II}}-\sqrt{k_{\mathrm{II}}^{2}-4 c_{\mathrm{I}} k_{\mathrm{I}}^{2}} \cos \left[2 \sqrt{c_{\mathrm{I}}}\left(x^{*}-x_{0}^{*}\right)\right]}{2 c_{\mathrm{I}}}} .
$$

Thus, in general the original wave amplitude $q$ admits a representation

$$
q(x)=\alpha\left(x^{*}\right) \rho(x)
$$

where $\alpha$ is an elliptic or trigonometric function, $x^{*}$ is the antiderivative of $\rho^{-2}(x)$ and the modulation $\rho(x)$ is driven by the classical Ermakov equation (3.5).

It is remarked that the above procedure with $\Lambda(x) \sim 1 / \rho^{6}$, but with $r(x) \neq 0$ in the wave representation (2.2), corresponds to a natural extension of that of Zhang et al [52] conducted in the context of a modulated NLS model descriptive of large amplitude Bloch wave propagation is optical lattices. That procedure, in turn, is embedded naturally in the reduction procedure presented earlier with modulation $\Lambda(x)=1 / \rho^{6} H(\rho / q)$ wherein $\rho(x)$ and wave amplitude $q(x)$ are governed by a Ermakov-Ray-Reid system.

\section{Transverse Wave Propagation in Hyperelastic Materials}

In a recent work by Destrade and Saccomandi [16] in the setting of nonlinear elastodynamics, a reduction to the classical Ermakov equation was obtained for the wave amplitude in transverse propagation in an incompressible hyperelastic material of neo-Hookean type subject to time-dependent biaxial stretch. In this hyperelastic context, the model coupled elastodynamic system is complex in nature, but not of variant NLS type unless rotatory effects are incorporated as in Ref. [14]. However, the algorithmic Ermakov-Ray-Reid reduction procedure as presented in the previous sections may nevertheless be adapted mutatis mutandis. Here, a reduction to a Ermakov type equation is obtained for Mooney-Rivlin materials via a wave packet ansatz analogous to that of (2.2) (but with the spatial and temporal roles interchanged). This is set in the context of transverse propagation in a class of higher order incompressible hyperelastic materials.

Thus, the class of shearing motions in the $Z$ direction $\mathbf{x}=\mathbf{x}(\mathbf{X}, t)$, with

$$
x=X+f(Z, t), \quad y=Y+g(Z, t), \quad z=Z,
$$

is considered. As usual, we denote by $\mathbf{F}=\partial \mathbf{x} / \partial \mathbf{X}$ the deformation gradient tensor, by $\mathbf{B}=\mathbf{F F}^{T}$ the left Cauchy-Green deformation tensor and by $I_{1}=\operatorname{tr} \mathbf{B}$ and $I_{2}=\operatorname{tr}\left(\mathbf{B}^{-1}\right)$ the first and second principal invariants. Since $\operatorname{det} \mathbf{F}=1$ for all $t$, the motion (4.1) is isochoric. 
The Cauchy stress tensor $\mathbf{T}$, derived from the strain-energy function $W=W\left(I_{1}, I_{2}\right)$ of an incompressible hyperelastic material, is

$$
\mathbf{T}=-p \mathbf{I}+2 \frac{\partial W}{\partial I_{1}} \mathbf{B}-2 \frac{\partial W}{\partial I_{2}} \mathbf{B}^{-1}
$$

where $p$ is a Lagrange multiplier introduced by the constraint of incompressibility, whence the nominal stress $\mathbf{S}=(\operatorname{det} \mathbf{F}) \mathbf{F}^{-1} \mathbf{T}$ is given by

$$
\mathbf{S}=-p \mathbf{F}^{-1}+2 W_{1} \mathbf{F}^{T}-2 W_{2} \mathbf{F}^{-1} \mathbf{B}^{-1} .
$$

The momentum equation

$$
\rho_{0} \frac{\partial^{2} \mathbf{x}}{\partial t^{2}}=\operatorname{Div} \mathbf{S}
$$

for the class of deformations (4.1) yields

$$
\begin{aligned}
& \rho_{0} f_{t t}=-p_{X}+2\left[\left(W_{1}+W_{2}\right) f_{Z}\right]_{Z}, \\
& \rho_{0} g_{t t}=-p_{Y}+2\left[\left(W_{1}+W_{2}\right) g_{Z}\right]_{Z}, \\
& 0=f_{Z} p_{X}+g_{Z} p_{Y}-p_{Z}+2\left[W_{1}-\left(1+f_{Z}^{2}+g_{Z}^{2}\right) W_{2}\right]_{Z},
\end{aligned}
$$

where subscripts indicate partial derivatives with respect to the indicated arguments.

On introduction of the shear strains $F:=f_{Z}$ and $G:=g_{Z}$, elimination of $p$ in (4.5) leads to a coupled nonlinear system for the strains $F$ and $G$, namely,

$$
\left\{\begin{array}{l}
\rho_{0} F_{t t}=(S F)_{Z Z}, \\
\rho_{0} G_{t t}=(S G)_{Z Z},
\end{array}\right.
$$

where the generalized shear modulus $S=2\left(W_{1}+W_{2}\right)$ is positive for all $Z$ and $t$ as a consequence of the Baker-Ericksen inequalities. Next, for the sake of completeness, from (4.5) and (4.6) the pressure field is found to be

$$
\begin{aligned}
& p=\left[(S F)_{Z}-\rho_{0} f_{t t}\right] x+\left[(S G)_{Z}-\rho_{0} g_{t t}\right] y \\
& +2 W_{1}-2\left(1+F^{2}+G^{2}\right) W_{2}+h(t),
\end{aligned}
$$

$h$ being an arbitrary function of time.

In terms of the quantity $\Theta:=F+i G$, the system (4.6) is conveniently encapsulated in the single complex equation

$$
\rho_{0} \Theta_{t t}=\left[S\left(|\Theta|^{2}\right) \Theta\right]_{Z Z} .
$$

A wave representation is here sought with

$$
\Theta(Z, t)=q(t) \exp [i(k Z+r(t))],
$$

whence, on substitution into (4.8), it is seen that

$$
\ddot{q}-q \dot{r}^{2}+\left(\frac{k^{2}}{\rho_{0}}\right) S\left(q^{2}\right) q=0,
$$


C. Rogers, G. Saccomandi, L. Vergori / Ermakov-Modulated Nonlinear Schrödinger Models

$$
2 \dot{r} \dot{q}+\ddot{r} q=0,
$$

where now a dot indicates a derivative with respect to time $t$. The latter relation (4.11) shows that (c.f. (2.5))

$$
\dot{r}=k_{\mathrm{I}} / q^{2},
$$

and substitution into (4.10) produces the nonlinear wave amplitude equation

$$
\ddot{q}+\left(\frac{k^{2}}{\rho_{0}}\right) S\left(q^{2}\right) q=\frac{k_{\mathrm{I}}^{2}}{q^{3}} .
$$

Here, we restrict attention to hyperelastic materials with strain energy potential of the type

$$
W\left(I_{1}, I_{2}\right)=\frac{1}{2} \sum_{m, n=0}^{2} C_{m n}\left(I_{1}-3\right)^{m}\left(I_{2}-3\right)^{n},
$$

where $C_{00}=0$ and $C_{m n}$ are material constants. For consistency of the model (4.14) with linear elasticity in the limit of small strains, it is necessary that

$$
C_{10}+C_{01}=\mu_{0}>0
$$

$\mu_{0}$ being the infinitesimal shear modulus, while requiring that (4.14) meets the the strong ellipticity condition implies that (see Ref. [46] for details)

$$
\mu_{n l} \equiv C_{20}+2 C_{11}+C_{02}>0 .
$$

For hyperelastic materials with strain energy given by (4.14), the generalized shear modulus $S$ is given by

$$
S=\mu_{0}+2 \mu_{n l} q^{2}
$$

and so the wave amplitude equation (4.13) becomes

$$
\ddot{q}+k^{2}\left(c^{2}+2 c_{n l}^{2} q^{2}\right) q=\frac{k_{\mathrm{I}}^{2}}{q^{3}},
$$

where

$$
c^{2}=\frac{\mu_{0}}{\rho_{0}}, \quad c_{n l}^{2}=\frac{\mu_{n l}}{\rho_{0}} .
$$

For a Mooney-Rivlin material $\left(C_{20}=C_{11}=C_{02}=0\right.$ in (4.14)), $c_{n l}=0$ and so equation (4.18) reduces to the classical Ermakov-type equation

$$
\ddot{q}+k^{2} c^{2} q=\frac{k_{\mathrm{I}}^{2}}{q^{3}} .
$$

The nonlinear superposition principle set down in Ref. [28] and subsequently constructed via Lie group methods in Ref. [35], as applied to (4.20) shows that

$$
q(t)=\sqrt{q_{0}^{2} \cos ^{2}(k c t)+\frac{q_{0} \dot{q}_{0}}{k c} \sin (2 k c t)+\frac{q_{0}^{2} \dot{q}_{0}^{2}+k_{\mathrm{I}}^{2}}{k^{2} c^{2} q_{0}^{2}} \sin ^{2}(k c t)},
$$




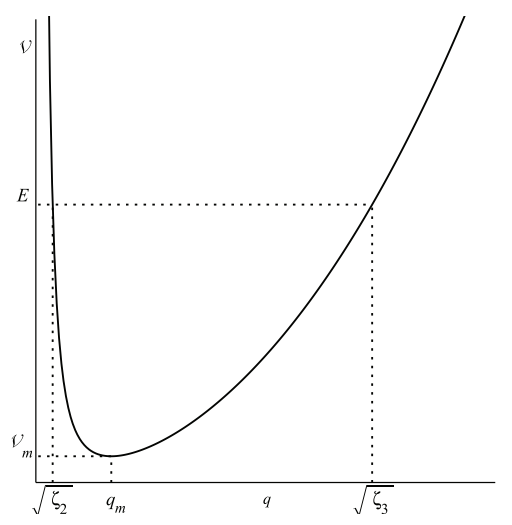

(a) $k_{\mathrm{I}} \neq 0$.

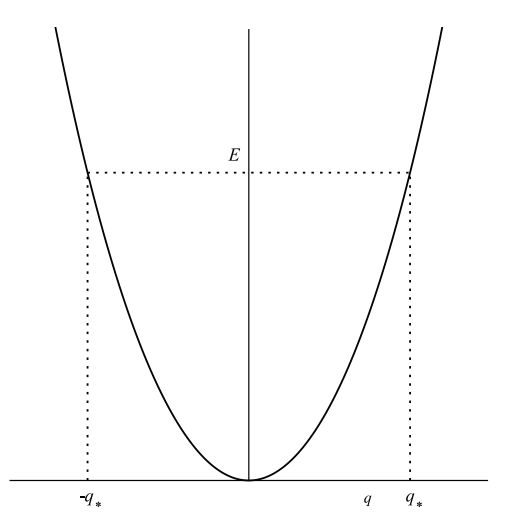

(b) $k_{\mathrm{I}}=0$.

Fig. 2. The different possible profiles of the function $\mathscr{V}$.

where $q_{0} \neq 0$ and $\dot{q}_{0}$ are integration constants. Equivalently, $q$ adopts the form

$$
q(t)=\sqrt{A+\sqrt{A^{2}-\frac{k_{\mathrm{I}}^{2}}{k^{2} c^{2}}} \cos \left[2 k c\left(t-t_{0}\right)\right]}
$$

where $A \geq\left|k_{\mathrm{I}}\right| /(|k| c)$ and $t_{0}$ are integration constants. Hence, in view of (4.12), the time-dependent phase $r$ is given by

$$
r(t)=\operatorname{sign}\left(\frac{k_{\mathrm{I}}}{k}\right)\left\{n \pi+\arctan \left[\sqrt{\frac{A-\sqrt{A^{2}-\frac{k_{\mathrm{I}}^{2}}{k^{2} c^{2}}}}{A+\sqrt{A^{2}-\frac{k_{\mathrm{I}}^{2}}{k^{2} c^{2}}}}} \tan \left[k c\left(t-t_{0}\right)-n \pi\right]\right]\right\}
$$

for $k c\left(t-t_{0}\right) \in\left[\frac{2 n-1}{2} \pi, \frac{2 n+1}{2} \pi\right]$ and $n \in \mathbb{Z}$.

In order to ascertain behavior predicted by (4.18) as one departs from the Mooney-Rivlin assumption corresponding to the Ermakov reduction with $\mu_{n l}=0$, we now consider the case $\mu_{n l}>0$. Equation (4.17) can be integrated once to obtain

$$
\dot{q}^{2}=E-k^{2}\left(c^{2}+c_{n l}^{2} q^{2}\right) q^{2}+\frac{k_{\mathrm{I}}}{q^{2}} \equiv E-\mathscr{V}(q)
$$

Obviously, the integrability of (4.24) depends on the positivity of the function $E-\mathscr{V}$ (Figure 2). If $k_{\mathrm{I}} \neq 0$ and $E \geq \mathscr{V}_{m} \equiv \mathscr{V}\left(q_{m}\right)$, where

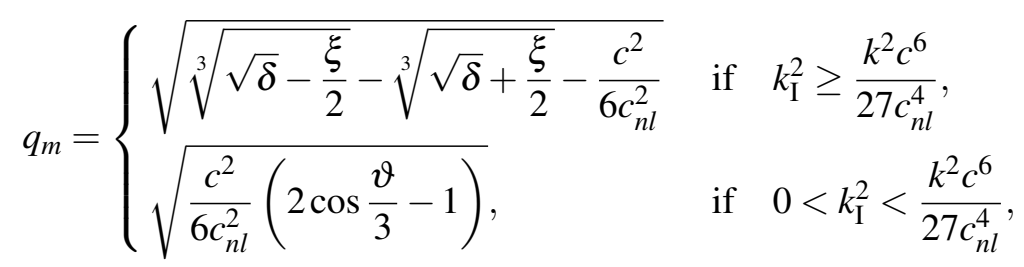

with

$$
\xi=\frac{c^{6}}{108 c_{n l}^{6}}-\frac{k_{\mathrm{I}}^{2}}{2 k^{2} c_{n l}^{2}}, \quad \delta=\frac{k_{\mathrm{I}}^{2}}{16 k^{4} c_{n l}^{4}}\left(k_{\mathrm{I}}^{2}-\frac{k^{2} c^{6}}{27 c_{n l}^{4}}\right), \quad \vartheta=\arccos \left(\frac{54 k_{\mathrm{I}}^{2} c_{n l}^{4}}{k^{2} c^{6}}-1\right),
$$


then the third order algebraic equation

$$
\zeta^{3}+\frac{c^{2}}{c_{n l}^{2}} \zeta^{2}-\frac{E}{k^{2} c_{n l}^{2}} \zeta+\frac{k_{\mathrm{I}}^{2}}{k^{2} c_{n l}^{2}}=0
$$

admits three real roots

$$
\zeta_{n}=\frac{c^{2}}{3 c_{n l}^{2}}\left(2 \sqrt{\frac{3 E c_{n l}^{2}}{k^{2} c^{4}}+1} \cos \frac{\phi+2 n \pi}{3}-1\right) \quad(n=1,2,3),
$$

where

$$
\phi=\arccos \left[-\frac{k\left(2 k^{2} c^{6}+9 E c^{2} c_{n l}^{2}+27 k_{\mathrm{I}}^{2} c_{n l}^{4}\right)}{2\left(k^{2} c^{4}+3 E c_{n l}^{2}\right)^{3 / 2}}\right] .
$$

These roots can be ordered as

$$
\zeta_{1}<0<\zeta_{2} \leq \zeta_{3}
$$

(It is worth noting that $\zeta_{2}=\zeta_{3}$ if and only if $E=\mathscr{V}_{m}$.)

Then, besides the solutions with constant amplitude

$$
\left\{\begin{array}{l}
q=\sqrt{\zeta_{n}}, \\
r(t)=\frac{k_{\mathrm{I}}}{\zeta_{n}}\left(t-t_{0}\right),
\end{array} \quad(n=2,3)\right.
$$

equations (4.12) and (4.24) admits a solution that can be written in terms of the Jacobi elliptic function sn and the incomplete elliptic integral of the third kind $\Pi$ :

$$
q(t)=\sqrt{\zeta_{3}-\left(\zeta_{3}-\zeta_{2}\right) \operatorname{sn}^{2}\left[\tau\left(t-t_{0}\right), \kappa\right]},
$$

and

$$
r(t)=\frac{k_{\mathrm{I}}}{\tau \zeta_{3}}\left\{2 n \Pi(1, v, \kappa)+\Pi\left(\operatorname{sn}\left[\tau\left(t-t_{0}\right)-n T, \kappa\right], v, \kappa\right)\right\}
$$

for $\tau\left(t-t_{0}\right) \in\left[\frac{(2 n-1) T}{2}, \frac{(2 n+1) T}{2}\right]$ and $n \in \mathbb{Z}$, where

$$
\tau=\sqrt{\zeta_{3}-\zeta_{1}} c_{n l} k, \quad \kappa=\sqrt{\frac{\zeta_{3}-\zeta_{2}}{\zeta_{3}-\zeta_{1}}}, \quad v=\frac{\zeta_{3}-\zeta_{2}}{\zeta_{3}}, \quad T=2 K(\kappa),
$$

$K$ being the complete elliptic integral of the first kind. The amplitude of this non-trivial solution oscillates between the constant solutions $q \equiv \sqrt{\zeta_{2}}$ and $q \equiv \sqrt{\zeta_{3}}$ (Figure 2(a)) with period $T / \tau$. Both the amplitude (4.32) and the time-dependent phase (4.33) tend respectively to (4.22) and (4.23), with $A=E /\left(2 k^{2} c^{2}\right)$, as $\mu_{n l}$ tends to zero (Figure 3). 


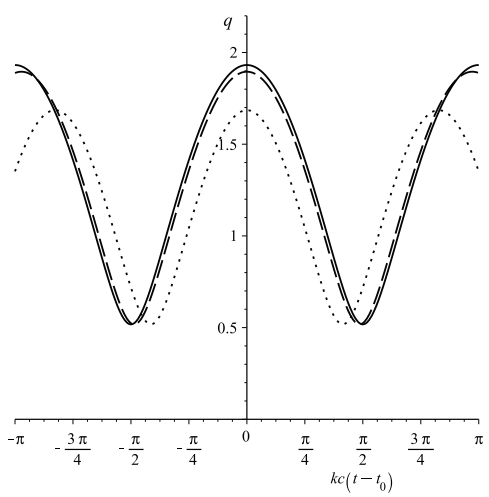

(a) Amplitude $q$.

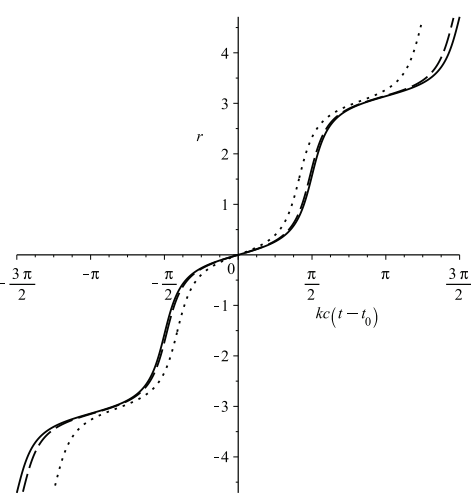

(b) Time-dependent phase $r$.

Fig. 3. Amplitude $q$ and phase $r$ for a Mooney-Rivlin material (solid line) and for a hyperelastic material with strainenergy function of the form (4.14) with $\mu_{n l} / \mu_{0}=0.01$ (dashed line) and $\mu_{n l} / \mu_{0}=0.1$ (dotted line). For the sake of illustration, the integration constants are taken $A=2, E=4 k^{2} c^{2}$ and $k_{\mathrm{I}}^{2}=k^{2} c^{2}$.

Finally, if $k_{\mathrm{I}}=0$ the solutions of equations (4.10)-(4.11) are standing waves with amplitude

$$
q(t)=q_{*} \operatorname{sn}\left[\frac{k c}{\sqrt{2 E} q_{*}}\left(t-t_{0}\right), i \frac{k c}{\sqrt{2 E}} q_{*}\right]
$$

where

$$
q_{*}=\frac{c}{\sqrt{2} c_{n l}} \sqrt{\sqrt{\frac{4 E c_{n l}^{2}}{k^{2} c^{4}}+1}-1},
$$

oscillating between the constant solutions $\pm q_{*}$ (Figure 2(b)) with period $\frac{4 \sqrt{2 E} q_{*}}{k c} K\left(i \frac{k c}{\sqrt{2 E}} q_{*}\right)$.

\section{Conclusion}

Here, integrable reductions of classes of spatially modulated nonlinear Schrödinger equation have been constructed subject to a novel connection between the modulation and Ermakov-Ray-Reid systems. The algorithmic procedure presented may be regarded as a natural extension of previous work in Ref. [52] in the context of Bloch wave and matter wave soliton propagation wherein the spatial modulation in the NLS model was driven by the classical Ermakov equation. It is noted that Ermakov-type reduction procedure may be readily adapted to the nonlinear elastodynamic system (4.6) as encapsulated in the complex representation (4.8).

\section{References}

[1] J. Abdullaev, A.S. Desyatnikov and E.A. Ostravoskaya, Suppression of collapse for matter waves with orbital angular momentum, J. Opt. 13 (2011) 064023.

[2] D.W. Barclay, T.B. Moodie and C. Rogers, Cylindrical impact waves in inhomogeneous Maxwellian visco-elastic media, Acta Mechanica 29 (1978) 93-117.

[3] J. Belmonte-Beita, V. M. Pérez-Garciá and V. Vekslechik, Lie symmetries and solitons in nonlinear systems with spatially inhomogeneous nonlinearities, Physical Review Letters 98 (2007) 064102. 
[4] J. Belmonte-Beita, V. M. Pérez-Garciá, V. Vekslechik and V.V. Konotop, Localized nonlinear waves in systems with time and space-modulated nonlinearities, Physical Review Letters 100 (2008) 164102.

[5] I. Bialynicki-Birula and J. Mycielski, Wave equations with logarithmic nonlinearities, Bull. Acad. Polon. Sci. 23 (1974) 461-466.

[6] I. Bialynicki-Birula and J. Mycielski, Nonlinear wave mechanics, Ann. Phys. 100 (1976) 62-93.

[7] I. Bialynicki-Birula and J. Mycielski, Gaussons: solitons of the logarithmic Schrödinger equation, Physica Scripta 20 (1979) 539-544.

[8] H.M. Cekirge and E. Varley, Large amplitude waves in bounded media: I Reflexion and transmission of large amplitude shockless pulses at an interface, Philos. Trans. Roy. Soc. London Ser. A 273 (1973) 261-313.

[9] K.W. Chow and C. Rogers, Localized and periodic wave patterns for a nonic nonlinear Schrödinger equation, Phys. Lett. A 377 (2013) 2546-2550.

[10] D.N. Christodoulidis, T.H. Coskun and R.I. Joseph, Incoherent spatial solitons in saturable nonlinear media, Opt. Lett. 22 (1997) 1080-1082.

[11] D.L. Clements and C. Rogers, On the Bergman operator method and anti-plane contact problems involving an inhomogeneous half-space, SIAM J. Appl. Math. 34 (1978) 764-773.

[12] D.L. Clements, C. Atkinson and C. Rogers, Antiplane crack problems for an inhomogeneous elastic material, Acta Mechanica 29 (1978) 199-211.

[13] F. Cornolti, M. Lucchesi and B. Zambon, Elliptic Gaussian beam self-focussing in nonlinear media, Opt. Commun. 75 (1990) 129-135.

[14] M. Destrade and G. Saccomandi, Some results on finite amplitude elastic waves propagating in rotating media, Acta Mechanica 173 (2004) 19-31.

[15] M. Destrade, M.D. Gilchrist and J.G. Murphy, Onset of non-linearity in the elastic bending of beams, ASME, J. Appl. Mech. 77 (2010) 061015.

[16] M. Destrade and G. Saccomandi, Transverse waves in nonlinearly elastic solids and the Milne-Pinney (or Ermakov) equation, Mathematics and Mechanics of Solids 16 (2011) 586-593.

[17] A.S. Desyatnikov, D. Buccoliero, M.R. Dennis and Y.S. Kivshar, Suppression of collapse for spiralling elliptic solitons, Phys. Rev. Lett. 104 (2010) 053902-1-053902-4.

[18] V.P. Ermakov, Second-order differential equations: conditions of complete integrability, Univ. Izy. Kiev 20 (1880) 1-25.

[19] A.M. Goncharenko, Y.A. Logvin, A.M. Samson, P.S. Shapovalov and S.I. Turovets, Ermakov Hamiltonian systems in nonlinear optics of elliptic Gaussian beams, Phys. Lett. A 160 (1991) 138-142.

[20] A.M. Goncharenko, Y.A. Logvin, A.M. Samson, P.S. Shapovalov, Rotating ellipsoidal gaussian beams in nonlinear media, Opt. Commun. 81 (1991) 225-230.

[21] A.M. Goncharenko, V.G. Kukushkin, Y.A. Logvin and A.M. Samson, Self-focussing of two orthogonally polarised light beams in a nonlinear medium, Opt. Quantum Electron 25 (1993) 97-104.

[22] C.R. Guiliano, J.H. Marburger and A. Yariv, Enhancement of self-focussing threshold in sapphire with elliptical beams, Appl. Phys. Lett. 21 (1972) 58-60.

[23] T. Hansson, D. Anderson and M. Lisak, Soliton interaction in logarithmically saturable media, Opt. Commun. 283 (2010) 318-322.

[24] F.C. Karal and J.B. Keller, Elastic wave propagation in homogeneous and inhomogeneous media, J. Acoust. Soc. Amer. 31 (1959) 694-705.

[25] K. Królikowski, D. Edmundson and O. Bang, Unified model for partially coherent solutions in logarithmically nonlinear media, Phys. Rev. E 61 (2000) 3122-3126.

[26] B.A. Malomed, Soliton Management in Periodic Systems (Springer, New York, 2006).

[27] T.B. Moodie, C. Rogers and D.L. Clements, Radial propagation of axial shear waves in an incompressible elastic material under finite deformation, Int. J. Engng. Sci. 14 (1976) 585-603.

[28] E. Pinney, The nonlinear differential equation $y^{\prime \prime}+p(x) y+c y^{-3}=0$, Proc Amer Math Soc 1 (1950) 681.

[29] J.R. Ray, Nonlinear superposition law for generalised Ermakov systems, Phys. Lett. A 78 (1980) 4-6.

[30] J.L. Reid and J.R. Ray, Ermakov systems, nonlinear superposition and solution of nonlinear equations of motion, J. Math. Phys. 21 (1980) 1583-1587. 
[31] C. Rogers, T.B. Moodie and D.L. Clements, Radial propagation of rotary shear waves in an initially stressed neo-Hookean material, J. de Mécanique 15 (1976) 595-614.

[32] C. Rogers, D.L. Clements and T.B. Moodie, Transient displacement and stress in non-homogeneous elastic shells, J. Elasticity 7 (1977) 171-184.

[33] C. Rogers, H.M. Cekirge and A. Askar, Electromagnetic wave propagation in non-linear dielectric media, Acta Mechanica 26 (1977) 59-73.

[34] C. Rogers and D.L. Clements, Bergman's integral operator method in inhomogeneous elasticity, Quart. Appl. Math. 36 (1978) 315-321.

[35] C. Rogers and U. Ramgulam, A nonlinear superposition principle and Lie group invariance: application in rotating shallow water theory, Int. J. Nonlinear Mech. 24 (1989) 229-236.

[36] C. Rogers, C. Hoenselaers and J.R. Ray, On 2+1-dimensional Ermakov systems, J. Phys. A: Math. Gen. 26 (1993) 2625-2633.

[37] C. Rogers and W.K. Schief, Multi-component Ermakov systems: structure and linearization, J. Math. Anal. Appl. 198 (1996) 194-220.

[38] C. Rogers, B. Malomed, K. Chow and H. An, Ermakov-Ray-Reid systems in nonlinear optics, J. Phys. A: Math. Theor. 43 (2010) 455214.

[39] C. Rogers and H. An, Ermakov-Ray-Reid systems in 2+1-dimensional rotating shallow water theory, Stud. Appl. Math. 125 (2010) 275-299.

[40] C. Rogers and H. An, On a 2+1-dimensional Madelung system with logarithmic and de Broglie-Bohm quantum potentials. Ermakov reduction, Physica Scripta 84 (2011) 045004.

[41] C. Rogers and W.K. Schief, On the integrability of a Hamiltonian reduction of a 2+1-dimensional nonisothermal rotating gas cloud system, Nonlinearity 24 (2011) 3165-3178.

[42] C. Rogers and W.K. Schief, The pulsrodon in 2+1-dimensional magnetogasdynamics. Hamiltonian structure and integrability theory, J. Math. Phys. 52 (2011) 083701.

[43] C. Rogers, B. Malomed and H. An, Ermakov-Ray-Reid reductions of variational approximations in nonlinear optics, Stud. Appl. Math. 129 (2012) 389-413.

[44] C. Rogers, B. Malomed and K.W. Chow, Invariants in a resonant derivative nonlinear Schrödinger model, J. Phys. A: Math. Theor. 45 (2012) 155205.

[45] C. Rogers, Gausson-type representations in nonlinear physics. Ermakov modulation, Physica Scripta 89 (2014) 105208.

[46] C. Rogers, G. Saccomandi and L. Vergori, Nonlinear Elastodynamics of Materials with Strong Ellipticity Condition: Carroll-Type Solutions, Wave Motion 56 (2015) 147-164.

[47] W.K. Schief, C. Rogers and A. Bassom, Ermakov systems with arbitrary order and dimension. Structure and linearisation, J. Phys. A: Math. Gen. 29 (1996) 903-911.

[48] W.K. Schief, H. An and C. Rogers, Universal and integrable aspects of an elliptic vortex representation in 2+1-dimensional magnetogasdynamics, Stud. Appl. Math. 130 (2013) 49-79.

[49] A.W. Snyder and J.D. Mitchell, Mighty morphing and spatial solitons and bullets, Opt. Lett. 22 (1997) 16-18.

[50] X.Y. Tang and P.K. Shukla, Solution of the one-dimensional spatially inhomogeneous cubic-quintic nonlinear Schródinger equation with external potential, Phys. Rev. A 76 (2007) 013612.

[51] W.G. Wagner, H.A. Haus and J.H. Marburger, Large-scale self-trapping of optical beams in the paraxial ray approximation, Phys. Rev. 175 (1968) 62-93.

[52] J.F. Zhang, Yi S. Li, J. Meng, L. Wo and B.A. Malomed, Matter-wave solitons and finite-amplitude Bloch waves in optical lattices with a spatially modulated nonlinearity, Phys. Rev. A 82 (2010) 033614.

[53] W.P. Zhong, M.R. Belić, B.A. Malomed and T. Huang, Solitary waves in the nonlinear Schrödinger equation with Hermite-Gaussian modulation of the local nonlinearity, Phys. Rev. E 84 (2011) 046611.

[54] W.P. Zhong, M.R. Belić and T. Huang, Solitary waves in the nonlinear Schrödinger equation with spatially modulated Bessel nonlinearity, J. Opt. Soc. Am B 30 (2013) 1276-1283. 